\title{
Axial-vector contributions in two-photon reactions: Pion transition form factor and deeply-virtual Compton scattering at NNLO in QCD
}

\author{
V. M. Braun, ${ }^{1}$ A. N. Manashov $\odot,{ }^{2,1,3}$ S. Moch, ${ }^{2}$ and J. Schönleber ${ }^{1}$ \\ ${ }^{1}$ Institut für Theoretische Physik, Universität Regensburg, D-93040 Regensburg, Germany \\ ${ }^{2}$ II. Institut für Theoretische Physik, Universität Hamburg D-22761 Hamburg, Germany \\ ${ }^{3}$ St. Petersburg Department of Steklov Mathematical Institute, 191023 St. Petersburg, Russia
}

(Received 8 June 2021; accepted 6 October 2021; published 5 November 2021)

\begin{abstract}
Using the approach based on conformal symmetry we calculate the two-loop coefficient function for the axial-vector contributions to two-photon processes in the $\overline{\mathrm{MS}}$ scheme. This is the last missing element for the complete next-to-next-to-leading order (NNLO) calculation of the pion transition form factor $\gamma^{*} \gamma \rightarrow \pi$ in perturbative QCD. The corresponding high-statistics measurement is planned by the Belle II collaboration and will allow one to put strong constraints on the pion light-cone distribution amplitude. The calculated NNLO corrections prove to be rather large and have to be taken into account. The same coefficient function determines the contribution of the axial-vector generalized parton distributions to deeply virtual Compton scattering (DVCS) which is investigated at the JLAB $12 \mathrm{GeV}$ accelerator, by COMPASS at CERN, and in the future will be studied at the Electron Ion Collider EIC.
\end{abstract}

DOI: 10.1103/PhysRevD.104.094007

A wealth of data on hard exclusive reactions from a new generation of experimental facilities will become available in the coming decade. These data are expected to have a very high precision and to provide a much deeper insight in the hadron structure as compared to the current knowledge. A pressing question is, however, whether hard exclusive hadronic reactions are under sufficient theoretical control to allow for fully quantitative predictions, which is highly relevant for all future high-intensity experiments. To give an example, the $\gamma^{*} \gamma \rightarrow \pi$ transition form factor is widely regarded as the "golden mode" that allows one to access the pion wave function at small transverse separations, usually referred to as the light-cone distribution amplitude (LCDA). The measurements of this form factor at spacelike momentum transfers in the interval $4-40 \mathrm{GeV}^{2}$ by the $B A B A R$ [1] and Belle [2] collaborations caused much excitement and a flurry of theoretical activity due to the unexpected large scaling violation observed by BABAR. At Belle II [3], the statistical uncertainty is expected to be reduced by a factor of 8 and the total systematic uncertainty is estimated to be at least 2 times smaller than that at Belle due to an improved trigger efficiency. As the result, a factor 3 to 5 times more precise measurements are possible in the high- $Q^{2}$ region.

Published by the American Physical Society under the terms of the Creative Commons Attribution 4.0 International license. Further distribution of this work must maintain attribution to the author(s) and the published article's title, journal citation, and DOI. Funded by SCOAP ${ }^{3}$.
Another example is provided by deeply virtual Compton scattering (DVCS) which is an important part of the physics program at the JLAB $12 \mathrm{GeV}$ upgrade [4], is measured also at CERN by COMPASS [5], and in the future will be studied at the EIC [6,7]. This reaction is the primary source of information on the generalized parton distributions (GPDs) of the nucleon (and, eventually, nuclei) which describe the correlation between parton's longitudinal momentum and its position in the transverse plane. Also in this case, the accuracy of the arriving and expected data is much higher as compared to the theoretical predictions available.

In both cases the theory framework is provided by collinear factorization and, despite obvious differences, there are some common elements. In particular the scale dependence of the LCDAs and GPDs is governed by similar equations, and the coefficient function $(\mathrm{CF})$ appearing in the factorized expression for the $\pi \gamma^{*} \gamma$ form factor in terms of the pion LCDA is the same as the CF of the axial GPD in DVCS. The corresponding calculations have to be advanced to next-to-next-to-leading order (NNLO) accuracy that has become standard in studies of inclusive processes and, since recently, also in semi-inclusive reactions in the framework of transverse momentum dependent factorization [8].

The new contribution of this work is a calculation of the two-loop CF for the flavor-non-singlet axial-vector contributions in processes with one real and one virtual photon. When combined with the three-loop anomalous dimensions calculated in [9], this result allows for the complete NNLO evaluation of the pion transition form factor and 
all flavor-non-singlet contributions to DVCS. In this letter we concentrate ourselves in the discussion of the numerical impact of the NNLO correction on $\pi \gamma^{*} \gamma$, since the DVCS observables are more complicated and require a dedicated study.

The pion transition form factor with one real and one virtual photon, $F\left(Q^{2}\right) \equiv F_{\pi \gamma^{*} \gamma}\left(Q^{2}\right)$, can be defined by the matrix element of the time-ordered product of two electromagnetic currents

$$
\begin{aligned}
& \int d^{4} y e^{i q y}\left\langle\pi^{0}(p)\left|T\left\{j_{\mu}^{\mathrm{em}}(y) j_{\nu}^{\mathrm{em}}(0)\right\}\right| 0\right\rangle \\
& =i e^{2} \varepsilon_{\mu \nu \alpha \beta} q^{\alpha} p^{\beta} F\left(Q^{2}\right)
\end{aligned}
$$

where $e$ is the electric charge, $p$ the pion momentum, and $j_{\mu}^{\mathrm{em}}=e_{u} \bar{u} \gamma_{\mu} u+e_{d} \bar{d} \gamma_{\mu} d+\cdots$. We will consider the spacelike form factor, $Q^{2}=-q^{2}>0$. The leading contribution $\mathcal{O}\left(1 / Q^{2}\right)$ to this form factor can be written in the factorized form $[10,11]$

$$
F\left(Q^{2}\right)=\frac{\sqrt{2} f_{\pi}}{6 Q^{2}} \int_{0}^{1} d z \mathrm{~T}(z, Q, \mu) \phi_{\pi}(z, \mu),
$$

where $f_{\pi}=131 \mathrm{MeV}$ denotes the pion decay constant, $\mu$ the factorization scale, $\mathrm{T}(z, Q, \mu)$ the $\mathrm{CF}$ and $\phi_{\pi}(z, \mu)$ the pion LCDA normalized as $\int_{0}^{1} d z \phi_{\pi}(z, \mu)=1$.

The same CF enters the axial-vector contributions to the DVCS amplitude $\mathcal{A}_{\mu \nu}=g_{\mu \nu}^{\perp} \mathcal{V}+\epsilon_{\mu \nu}^{\perp} \mathcal{A}$

$$
\mathcal{A}\left(\xi, Q^{2}\right)=\frac{1}{2} \sum_{q} e_{q}^{2} \int_{-1}^{1} \frac{d x}{\xi} \mathrm{T}\left(\frac{\xi-x}{2 \xi}, Q, \mu\right) \tilde{F}_{q}(x, \xi, t, \mu),
$$

where $\tilde{F}_{q}(x, \xi, t, \mu)$ is the axial-vector GPD and $\xi$ is the skewedness parameter; see [12,13] for details. Here it is assumed that $\mathrm{T}\left(\frac{\xi-x}{2 \xi}, Q^{2}, \mu\right)$ is continued analytically to the $|x / \xi|>1$ region using the $\xi \rightarrow \xi-i \epsilon$ prescription.

The CF can be expanded in powers of the strong coupling, $a_{s}=\alpha_{s}(\mu) / 4 \pi$,

$$
\mathrm{T}=\mathrm{T}^{(0)}+a_{s} \mathrm{~T}^{(1)}+a_{s}^{2} \mathrm{~T}^{(2)}+\ldots
$$

The first two terms in this series are well known $[11,14-16]$ :

$$
\mathrm{T}^{(0)}(z)=1 / z+1 / \bar{z}
$$

$$
\begin{aligned}
\mathrm{T}^{(1)}(z, Q, \mu)= & C_{F} \frac{1}{z}\left[\ln ^{2} z-\frac{z \ln z}{\bar{z}}-9+(3+2 \ln z) \mathrm{L}\right] \\
& +(z \leftrightarrow \bar{z}),
\end{aligned}
$$

where $\bar{z}=1-z$ and $\mathrm{L}=\ln \left(Q^{2} / \mu^{2}\right)$. The two-loop correction $\mathrm{T}^{(2)}$ is the subject of this work. It can be decomposed in the contributions of three color structures:

$$
\mathrm{T}^{(2)}=C_{F}^{2} \mathrm{~T}_{\mathrm{P}}^{(2)}+\frac{C_{F}}{N_{c}} \mathrm{~T}_{\mathrm{NP}}^{(2)}+\beta_{0} C_{F} \mathrm{~T}_{\beta}^{(2)},
$$

where $C_{F}=\left(N_{c}^{2}-1\right) / 2 N_{c}$ and $\beta_{0}=11 / 3 N_{c}-2 / 3 n_{f}$ in a $\mathrm{SU}\left(N_{c}\right)$ gauge theory. We obtain (for $\mu=Q$ )

$$
\begin{aligned}
\mathrm{T}_{\mathrm{P}}^{(2)}= & \left\{\frac{2}{z}\left(6 \mathrm{H}_{0000}-\mathrm{H}_{1000}-2 \mathrm{H}_{200}-\mathrm{H}_{1100}-\mathrm{H}_{120}-\mathrm{H}_{210}+\mathrm{H}_{1110}\right)+\frac{2}{\bar{z}} \mathrm{H}_{000}-\frac{8}{z} \mathrm{H}_{100}+\frac{2}{\bar{z}} \mathrm{H}_{20}+\frac{4}{z} \mathrm{H}_{110}\right. \\
& -\left(\frac{11}{\bar{z}}+\frac{38}{3 z}\right) \mathrm{H}_{00}+\frac{34}{3 z} \mathrm{H}_{10}+\frac{2}{z} \zeta_{2}\left(\mathrm{H}_{11}-\mathrm{H}_{2}-\mathrm{H}_{10}-4 \mathrm{H}_{00}\right)-\frac{1}{\bar{z}}\left(2 \zeta_{2}-4 \zeta_{3}-\frac{71}{2}\right) \mathrm{H}_{0} \\
& \left.+\frac{1}{z}\left(6 \zeta_{2}+32 \zeta_{3}-\frac{64}{9}\right) \mathrm{H}_{0}+\frac{1}{z}\left(\frac{701}{24}+\frac{34}{3} \zeta_{2}+43 \zeta_{3}+3 \zeta_{2}^{2}\right)\right\}+(z \leftrightarrow \bar{z}), \\
\mathrm{T}_{\mathrm{NP}}^{(2)}= & \left\{12\left(\mathrm{H}_{20}+\mathrm{H}_{110}-\frac{1}{z} \mathrm{H}_{10}-\frac{1}{\bar{z}} \mathrm{H}_{0}-\zeta_{3}\right)-\frac{6}{z} \mathrm{H}_{200}-\left(\frac{2}{\bar{z}}+\frac{4}{z}\right)\left(\mathrm{H}_{30}+\mathrm{H}_{210}+\mathrm{H}_{31}\right)+\left(\frac{2}{\bar{z}}+\frac{8}{z}\right) \mathrm{H}_{4}\right. \\
+ & \left(\frac{4}{\bar{z}}+\frac{6}{z}\right) \mathrm{H}_{22}+\frac{6}{\bar{z}}\left(\mathrm{H}_{3}-\mathrm{H}_{20}\right)+\left[\frac{2}{\bar{z}}\left(1-\zeta_{2}\right)+\frac{4}{z}\left(\frac{2}{3}-\zeta_{2}\right)\right] \mathrm{H}_{00}-\left[\frac{4}{\bar{z}}\left(\zeta_{2}-1\right)-\frac{1}{\bar{z}}\left(\frac{38}{3}-2 \zeta_{2}\right)\right] \mathrm{H}_{2} \\
+ & {\left.\left[\frac{1}{z}\left(14 \zeta_{3}-\frac{32}{9}\right)+\frac{1}{\bar{z}}\left(1-6 \zeta_{2}+6 \zeta_{3}\right)\right] \mathrm{H}_{0}-\frac{1}{z}\left(3 \zeta_{2}^{2}+20 \zeta_{2}-36 \zeta_{3}+\frac{73}{12}\right)\right\}+(z \leftrightarrow \bar{z}), }
\end{aligned}
$$




$$
\begin{aligned}
\mathrm{T}_{\beta}^{(2)}= & \left\{-\frac{2}{z} \mathrm{H}_{000}+\frac{2}{z} \mathrm{H}_{100}-\frac{1}{z} \mathrm{H}_{110}+\left(\frac{10}{3 z}-\frac{1}{\bar{z}}\right) \mathrm{H}_{00}-\frac{14}{3 z} \mathrm{H}_{10}+\left[\frac{1}{\bar{z}}\left(\frac{1}{2}+\zeta_{2}\right)-\frac{19}{9 z}\right] \mathrm{H}_{0}\right. \\
& \left.-\frac{1}{z}\left(\zeta_{3}+\frac{14}{3} \zeta_{2}+\frac{457}{24}\right)\right\}+(z \leftrightarrow \bar{z}),
\end{aligned}
$$

where $\mathrm{H}_{\vec{m}}=\mathrm{H}_{\vec{m}}(z)$ are harmonic polylogarithms [17]. Our result for $\mathrm{T}_{\beta}^{(2)}$ is in agreement with Ref. [18]. The expressions for $\mathrm{T}_{\mathrm{P}}^{(2)}$ (planar), $\mathrm{T}_{\mathrm{NP}}^{(2)}$ (nonplanar) are new results. Complete results for $\mathrm{T}^{(2)}(z, \mu)$ in Mathematica format are presented in the ancillary file (see supplemental material [19]).

Our method of calculation is based on using conformal symmetry of QCD at critical coupling in $d=4-2 \epsilon$ dimensions to relate the $\mathrm{CF}$ in question to the well-known two-loop axial-vector $\mathrm{CF}$ in deep-inelastic scattering [20,21]. A similar idea was used before in Ref. [22] where the NNLO CF was calculated in a special, conformal renormalization scheme. Our technique is explained in detail in Ref. [23] where the vector CF was calculated by the same method. The generalization to axial-vector operators is considered in Ref. [24]. Thus in what follows we will only outline the main steps and omit technical details.

The starting point is that QCD at the Wilson-Fischer critical point at noninteger $d=4-2 \epsilon^{*}$ space-time dimensions, $\epsilon^{*}=-\beta_{0} a_{s}-\beta_{1} a_{s}^{2}+\cdots$, is a conformal theory. The strategy is to calculate the CF in conformal QCD, and go over to the "physical" limit $\epsilon \rightarrow 0$ at the very end, by adding a term $\sim \epsilon^{*}$ :

$$
\mathrm{T}=\mathrm{T}_{*}+\epsilon^{*} \Delta \mathrm{T}
$$

The extra term $\Delta \mathrm{T}$ requires a one-loop calculation and only affects $\mathrm{T}_{\beta}^{(2)}$.

We define axial-vector operators in $d=4-2 \epsilon$ dimensions using a variant of Larin's scheme [25] for the $\gamma_{5}$ matrix; see ref. [24]. In $d=4$ the axial-vector operators in this scheme can be rotated to the $\overline{\mathrm{MS}}$ scheme which is defined by the condition that the vector- and axial-vector flavor-non-singlet operators satisfy the same evolution equation.

The $\mathrm{CF}$ in the $\overline{\mathrm{MS}}$ scheme can be written in the form [23],

$$
\mathrm{T}_{*}=\mathrm{T}^{(0)} \otimes K \otimes e^{\mathbb{X}} \otimes \mathcal{U}^{-1},
$$

where $\mathrm{T}^{(0)}$ is the tree-level CF, $e^{\rtimes}$ takes into account the conformal anomaly $[24,26], \mathcal{U}$ is the rotation operator from Larin's scheme to the $\overline{\mathrm{MS}}$ scheme [24], and $K$ is a certain SL(2)-invariant operator (i.e., $K$ commutes with the generators of conformal transformations). The eigenvalues of $K$ can be related to the moments of the axial vector $\mathrm{CF}$ in deep-inelastic scattering $[20,21]$ up to some additional factors, cf. Eq. (3.59) in Ref. [23]. We have calculated these eigenvalues in terms of harmonic sums using computer algebra packages [27-29]. The result satisfies the reciprocity relation [30,31]: the asymptotic expansion of the eigenvalues of $K$ at large spin $N$ is symmetric under the substitution $N \rightarrow-N-1$. This property provides a nontrivial test of the calculation.

Any SL(2) invariant operator is uniquely defined by its spectrum. Thus $K$ can be restored, and it remains to do the convolutions in Eq. (12) to obtain the final result. A direct evaluation of the convolution in momentum space at two loops is very cumbersome, but it can be bypassed, as explained in Ref. [23], using a position-space representation at the intermediate step. In this way one ends up with much simpler integrals that we have calculated with the help of the HyperInt package [32].

The ratios of the NLO/LO, NNLO/LO and NNLO/NLO CFs at the scale $Q^{2}=\mu^{2}=4 \mathrm{GeV}^{2}$ are plotted in Fig. 1 . One sees that the two-loop $\mathcal{O}\left(a_{s}^{2}\right)$ correction has the same sign and is roughly factor two smaller as compared to the one-loop contribution in the bulk of the $z$ region. The largest contribution to $\mathrm{T}^{(2)}$ comes from $\mathrm{T}_{\beta}$ except for the endpoint regions where the leading effect is due to the Sudakov-like double-logarithmic corrections

$$
\mathrm{T}\left(z, a_{s}\right) \underset{z \mapsto 0}{\simeq} \frac{1}{z}\left(1+C_{F} a_{s} \ln ^{2} z+\frac{\left(C_{F} a_{S}\right)^{2}}{2} \ln ^{4} z+\ldots\right) .
$$

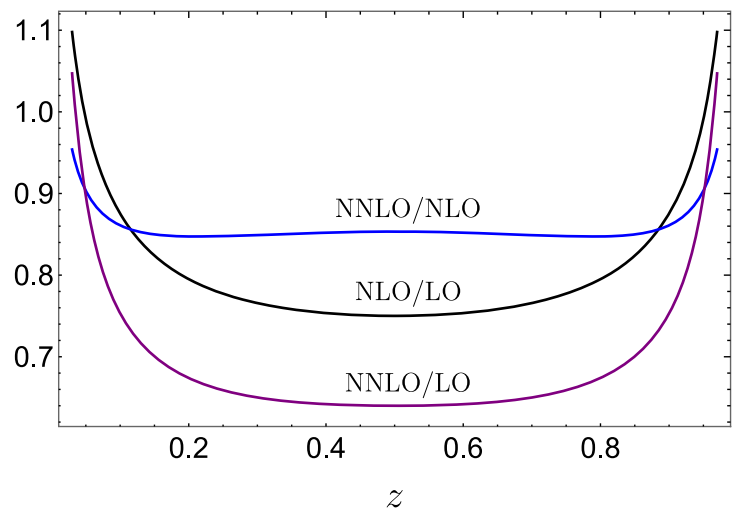

FIG. 1. The ratios $T_{\mathrm{NLO}} / T_{\mathrm{LO}}, T_{\mathrm{NNLO}} / T_{\mathrm{LO}}$, and $T_{\mathrm{NNLO}} / T_{\mathrm{NLO}}$ at the scale $Q^{2}=\mu^{2}=4 \mathrm{GeV}^{2}$. Here $T_{\mathrm{LO}}=T^{(0)}, T_{\mathrm{NLO}}=T^{(0)}+$ $a_{s} T^{(1)}$ and $T_{\mathrm{NNLO}}=T^{(0)}+a_{s} T^{(1)}+a_{s}^{2} T^{(2)}$ are the CFs defined in (4) truncated at the first (tree level), second (one-loop) and third (two-loop) terms, respectively. For this calculation we used $\alpha_{s}(\mu=2 \mathrm{GeV})=0.3009$ and $n_{f}=4$. 


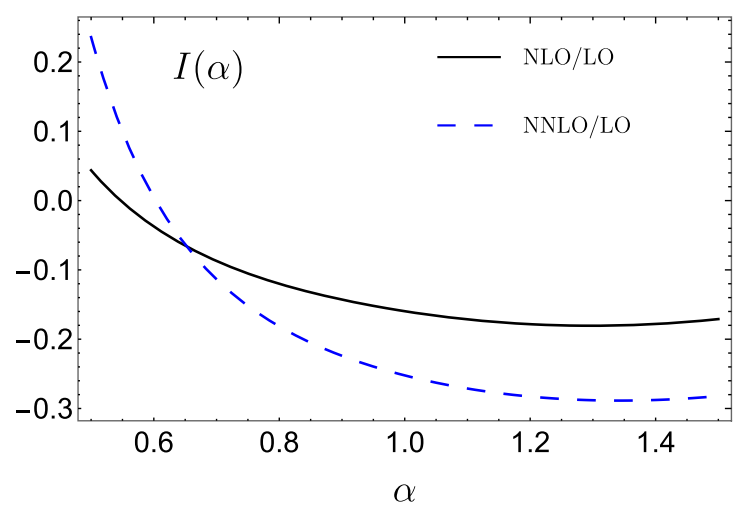

FIG. 2. The NNLO/LO and NLO/LO ratios for the integral $I(\alpha)$ in Eq. (13) as a function of $\alpha$.

The series likely exponentiates resulting in $\mathrm{T}\left(z, a_{s}\right) \sim$ $z^{-1+a_{s} C_{F} \ln z}$.

Note that also the sign of the correction changes, so that the resulting effect on physics observables will depend strongly on the behavior of the parton distributions at the endpoints. As an illustration, we show in Fig. 2 the ratios $I_{\mathrm{NLO}} / I_{\mathrm{LO}}$ and $I_{\mathrm{NNLO}} / I_{\mathrm{LO}}$ for the integral

$$
I(\alpha)=\int_{0}^{1} d z z^{\alpha} \bar{z}^{\alpha} \mathrm{T}\left(z, Q^{2}, \mu^{2}=Q^{2}\right)
$$

as a function of $\alpha$ in the interval $0.5<\alpha<1.5$.

The pion LCDA is usually represented by the expansion in terms of Gegenbauer polynomials which are eigenfunctions of the one-loop evolution equations

$$
\phi_{\pi}(z, \mu)=6 z \bar{z} \sum_{n=0,2, \ldots} a_{n}(\mu) C_{n}^{3 / 2}(2 z-1) .
$$

Here $a_{0}=1$ is fixed by the normalization condition, and $a_{2}$ is known from lattice calculations. We use the latest result [33]

$$
a_{2}\left(\mu_{0}=2 \mathrm{GeV}\right)=0.116_{-20}^{+19} .
$$

For the coefficients $a_{n}$ with $n>2$ there exist only very weak constraints. In order to estimate their influence, following [33], we consider two models for the pion LCDA (at the scale $\left.\mu_{0}=2 \mathrm{GeV}\right)$ :

$\phi_{\pi}^{(I)}\left(z, \mu_{0}\right)=6 z(1-z)\left[1+a_{2}\left(\mu_{0}\right) C^{3 / 2}(2 z-1)\right]$,

$\phi_{\pi}^{(I I)}\left(z, \mu_{0}\right)=B(1+\alpha, 1+\alpha) z^{\alpha}(1-z)^{\alpha}$,

where the parameter $\alpha$ in the second model is adjusted to reproduce the same value of the second Gegenbauer coefficient $a_{2}$ as in Eq. (15); $B(a, b)$ is Euler's $\beta$-function.

Using the expansion in Eq. (14) the $\pi \gamma^{*} \gamma$ form factor to leading-twist accuracy is given by

$$
Q^{2} F\left(Q^{2}\right)=\sqrt{2} f_{\pi} \sum_{n=0,2, \ldots} a_{n}(\mu) c_{n}(\mu, \mathrm{L}),
$$

where the coefficients

$$
c_{n}(\mu, \mathrm{L})=1+a_{s} c_{n}^{(1)}(\mu, \mathrm{L})+a_{s}^{2} c_{n}^{(2)}(\mu, \mathrm{L})+\ldots
$$

are given by the Gegenbauer moments of the $\mathrm{CF} \mathrm{T}(z, Q, \mu)$ in Eq. (4). The one-loop coefficients read

$$
\begin{aligned}
c_{n}^{(1)}= & C_{F}\left\{4 S_{1}^{2}(n+1)-\frac{4 S_{1}(n+1)-3}{(n+1)(n+2)}+\frac{2}{(n+1)^{2}(n+2)^{2}}\right. \\
& \left.-9-2\left[S_{1}(n+2)+S_{1}(n)-\frac{3}{2}\right] \mathrm{L}\right\},
\end{aligned}
$$

where $S_{1}(n)$ are the standard harmonic sums, and the first few two-loop coefficients are given by

$$
\begin{aligned}
& c_{0}^{(2)}=-197.40+9.66 n_{f}+\mathrm{L}\left(68.50-2.92 n_{f}\right), \\
& c_{2}^{(2)}=95.97-17.44 n_{f}+\mathrm{L}\left(-178.25+10.9 n_{f}\right)+\mathrm{L}^{2}\left(45.99-1.85 n_{f}\right), \\
& c_{4}^{(2)}=526.97-43.81 n_{f}+\mathrm{L}\left(-417.06+20.42 n_{f}\right)+\mathrm{L}^{2}\left(77.20-2.67 n_{f}\right), \\
& c_{6}^{(2)}=986.91-67.14 n_{f}+\mathrm{L}\left(-663.97+27.73 n_{f}\right)+\mathrm{L}^{2}\left(101.63-3.26 n_{f}\right) .
\end{aligned}
$$

In these expressions we included the logarithmic contributions, $\mathrm{L}=\ln Q^{2} / \mu^{2}$, to allow for the study of the factorization scale dependence. Note that the radiative corrections (both one-loop and two-loop) to the leading contribution $n=0$ (asymptotic LCDA) are negative, whereas the corrections to the contributions of higher moments are positive and increase with $n$. Thus the radiative corrections to the form factor in general amplify the contributions of higher-order Gegenbauer polynomials at high photon virtualities.

The results for the NNLO vs NLO calculation of the $\pi \gamma^{*} \gamma$ form factor for the two models of the pion LCDA in Eq. (16) are shown in Fig. 3. In this calculation we set $\mu=Q$ and use the evolution equations both at NLO and NNLO [9], to calculate the pion LCDA (16) at this scale. It is seen that the model dependence is comparable in size 


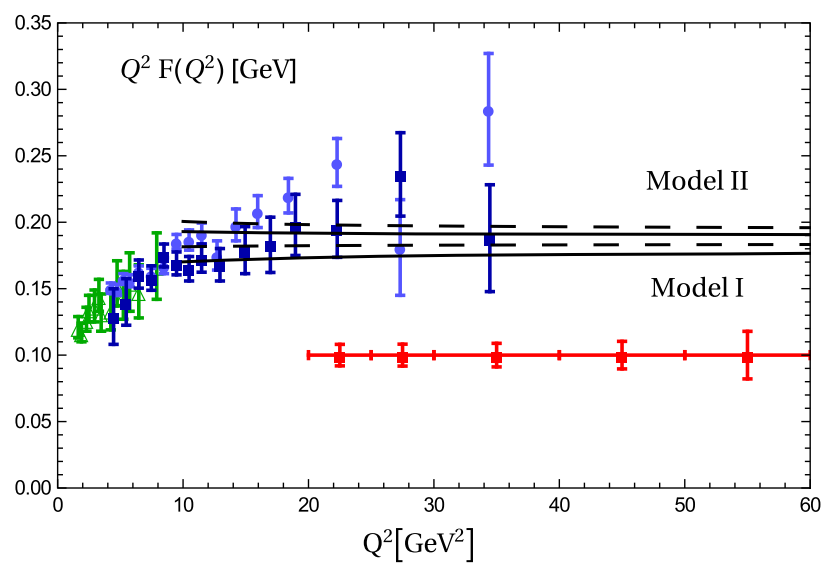

FIG. 3. The $\pi \gamma^{*} \gamma$ form factor at the NNLO (solid curves) and NLO (dashed curves) in QCD perturbation theory for the two models of the pion LCDA in Eq. (16). The experimental data are from CLEO [34] (green, open triangles), BABAR [1] (light blue, circles) and Belle [2] (dark blue, squares). In addition, the expectation for the error bars achievable at Belle II [3] is shown in red. The central value for the red boxes is arbitrary.

with the projected accuracy of the Belle II measurements. The NNLO correction is about a half of the model difference and has to be taken into account. We conclude that the NNLO accuracy is mandatory to constrain the pion LCDA from the analysis of theory predictions with the expected data. This, in turn, will have important consequences on the accuracy of QCD predictions in B-decays and other hard processes with energetic pions in the final state.

As already mentioned, the same CF enters the NNLO calculation of the contribution of the axial-vector GPD to DVCS. The DVCS amplitude (3) is a complex-valued function. Following [35] we show in Fig. 4 the size of the NNLO two-loop correction for the absolute value and the phase difference of the corresponding Compton form factor at $Q^{2}=\mu^{2}=4 \mathrm{GeV}^{2}$,

$$
\tilde{\mathcal{H}}(\xi)=R(\xi) e^{i \Phi(\xi)},
$$

for the simplest ansatz for the GPD $\tilde{H}$, see Eq. (3.330) in [13], normalized in the forward limit to the polarized quark density $f(\beta) \sim \beta^{1 / 2}(1-\beta)^{4}$. The results are qualitatively very similar to the vector amplitude considered in Ref. [23]: The two-loop correction is large for the absolute value of the Compton form factor and small for the phase. A more detailed study including the scale dependence is premature at this time, as the axial-vector GPD is practically unknown. This task will become important in the future to analyze the forthcoming JLAB-12 and, later, EIC data.

In summary, we have used innovative computational approaches based on conformal symmetry to advance the
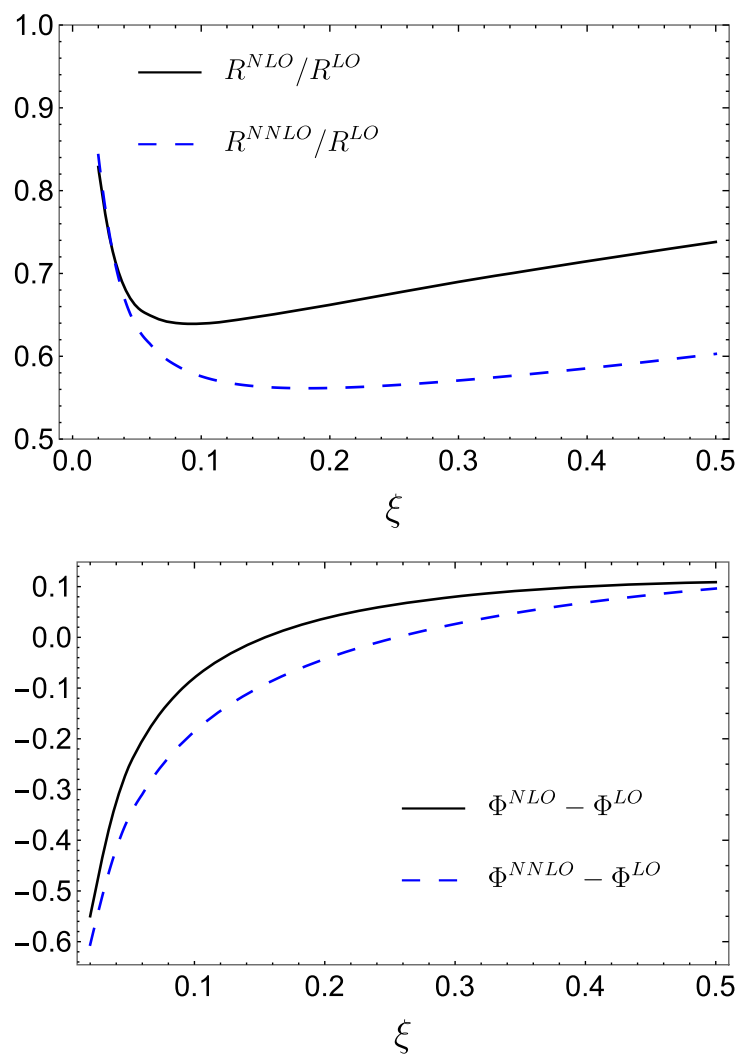

FIG. 4. The axial-vector Compton form factor $\mathcal{H}(\xi)$ in Eq. (21), calculated to NNLO and NLO accuracy. Shown are the ratios for the absolute values (top panel) and the phase differences (bottom panel), both with respect to the tree-level.

theoretical predictions for hard exclusive reactions to NNLO in QCD, which is the level of accuracy required by the precision of experimental data. We expect our results to have a broad range of applications in the analyses of data from current and future high-intensity, medium energy experiments.

This study was supported by DFG Research Unit FOR 2926, Grant No. 40824754, DFG Grants No. MO 1801/4-1, No. KN 365/13-1, and RSF Project No. 19-11-00131. The authors are grateful to Yao Ji for communication on an independent calculation of the same CF by another method. We thank Sadaharu Uehara for providing us with the estimates for the expected accuracy of the Belle II experiment. Our special thanks are due to A. V. Pimikov for pointing out a misprint in the ancillary file.

Note Added.-Our result for the CF in Eqs. (8)-(10) agrees with an independent calculation of the same CF using a different technique [36], cf. Eqs. (26)-(29). It is easy to check that the analytic expressions in this work and in [36] coincide identically. 
[1] B. Aubert et al. (BABAR Collaboration), Phys. Rev. D 80, 052002 (2009).

[2] S. Uehara et al. (Belle Collaboration), Phys. Rev. D 86, 092007 (2012).

[3] W. Altmannshofer et al. (Belle-II Collaboration), Prog. Theor. Exp. Phys. (2019), 123C01; (2020), 029201(E).

[4] J. Dudek et al., Eur. Phys. J. A 48, 187 (2012).

[5] R. Akhunzyanov et al. (COMPASS Collaboration), Phys. Lett. B 793, 188 (2019).

[6] A. Accardi et al., Eur. Phys. J. A 52, 268 (2016).

[7] R. Abdul Khalek et al., arXiv:2103.05419.

[8] D. Gutierrez-Reyes, I. Scimemi, and A. Vladimirov, J. High Energy Phys. 07 (2018) 172.

[9] V. M. Braun, A. N. Manashov, S. Moch, and M. Strohmaier, J. High Energy Phys. 06 (2017) 037.

[10] G. P. Lepage and S. J. Brodsky, Phys. Lett. 87B, 359 (1979).

[11] G. P. Lepage and S. J. Brodsky, Phys. Rev. D 22, 2157 (1980).

[12] M. Diehl, Phys. Rep. 388, 41 (2003).

[13] A. V. Belitsky and A. V. Radyushkin, Phys. Rep. 418, 1 (2005).

[14] F. del Aguila and M. K. Chase, Nucl. Phys. B193, 517 (1981).

[15] E. Braaten, Phys. Rev. D 28, 524 (1983).

[16] E. P. Kadantseva, S. V. Mikhailov, and A. V. Radyushkin, Yad. Fiz. 44, 507 (1986).

[17] E. Remiddi and J. A. M. Vermaseren, Int. J. Mod. Phys. A 15, 725 (2000).

[18] B. Melic, B. Nizic, and K. Passek, Phys. Rev. D 65, 053020 (2002).
[19] See Supplemental Material at http://link.aps.org/supplemental/ 10.1103/PhysRevD.104.094007 for the complete results for the two-loop CF in Mathematica format.

[20] E. B. Zijlstra and W. L. van Neerven, Nucl. Phys. B417, 61 (1994); B426, 245(E) (1994); 501, 599(E) (1997); 773, 105(E) (2007).

[21] S. Moch and J. A. M. Vermaseren, Nucl. Phys. B573, 853 (2000).

[22] B. Melic, D. Müller, and K. Passek-Kumericki, Phys. Rev. D 68, 014013 (2003).

[23] V. M. Braun, A. N. Manashov, S. Moch, and J. Schoenleber, J. High Energy Phys. 09 (2020) 117.

[24] V. M. Braun, A. N. Manashov, S. Moch, and M. Strohmaier, Phys. Rev. D 103, 094018 (2021).

[25] S. A. Larin, Phys. Lett. B 303, 113 (1993).

[26] V. M. Braun, A. N. Manashov, S. Moch, and M. Strohmaier, J. High Energy Phys. 03 (2016) 142.

[27] J. Vermaseren, Int. J. Mod. Phys. A 14, 2037 (1999).

[28] J. Ablinger, Ph.D. thesis, Linz U., 2009.

[29] J. Ablinger, Proc. Sci., LL2014 (2014) 019 [arXiv:1407.6180].

[30] B. Basso and G. P. Korchemsky, Nucl. Phys. B775, 1 (2007).

[31] L. F. Alday, A. Bissi, and T. Lukowski, J. High Energy Phys. 11 (2015) 101.

[32] E. Panzer, Comput. Phys. Commun. 188, 148 (2015).

[33] G. S. Bali et al. (RQCD Collaboration), J. High Energy Phys. 08 (2019) 065; 11 (2020) 037(A).

[34] J. Gronberg et al. (CLEO Collaboration), Phys. Rev. D 57, 33 (1998).

[35] K. Kumericki, D. Müller, and K. Passek-Kumericki, Nucl. Phys. B794, 244 (2008).

[36] J. Gao, T. Huber, Y. Ji, and Y.-M. Wang, arXiv:2106.01390. 